\title{
A narrative review of surgical management of gastrointestinal stromal tumors
}

\author{
Iwona Kalinowska, Marcin Zdzienicki, Jacek Skoczylas, Piotr Rutkowski
}

Maria Sklodowska-Curie National Research Institute of Oncology. Department of Soft Tissue/Bone Sarcoma and Melanoma, Warsaw, Poland

Contributions: (I) Conception and design: I Kalinowska, P Rutkowski; (II) Administrative support: All authors; (III) Provision of study material or patients: P Rutkowski, M Zdzienicki, I Kalinowska; (IV) Collection and assembly of data: I Kalinowska, P Rutkowski, J Skoczylas; (V) Data analysis and interpretation: All authors; (VI) Manuscript writing: All authors; (VII) Final approval of manuscript: All authors.

Correspondence to: Piotr Rutkowski, MD, PhD. Maria Sklodowska-Curie National Research Institute of Oncology, Department of Soft Tissue/Bone Sarcoma and Melanoma, Roentgena Str. 5 02-781, Warsaw, Poland. Email: piotr.rutkowski@pib-nio.pl.

\begin{abstract}
Radical surgical treatment remains the basic method of treatment of patients with localized resectable gastrointestinal stromal tumors (GIST), which allows achieving a permanent remission of the disease in over $50 \%$ of cases. The assessment of the risk of disease recurrence after radical surgery is used in clinical practice to determine the prognosis and plan multimodal management of an individual patient. Moreover, at present, the role of surgery in this entity is not limited to resection of the primary tumor but also in initially unresectable or metastatic tumors in combination with systemic targeted therapy. In this review, we mainly focus on the role of surgery and the treatment of primary GIST. The surgical treatment role including the technical aspects of therapy for each lesion's location, taking into account the latest advances in laparoscopic and robotic surgery techniques. When discussing the surgical aspects of GIST treatment, we cannot neglect the importance of surgery in some cases in the diagnosis of these tumors. We also have presented information about the important role of surgical margins and touched upon some points of surgical aspects of treatment after combination therapy with tyrosine kinase inhibitors (TKIs) in locally advanced and metastatic GIST. We systematized the current knowledge and guidelines on the surgical treatment of GIST, which can be recognized as a compendium for treatment in clinical practice.
\end{abstract}

Keywords: Gastrointestinal stromal tumor (GIST); surgery; laparoscopy; robotic surgery; combined therapy

Received: 24 February 2021; Accepted: 02 August 2021; Published: 30 September 2021.

doi: 10.21037 /gist-21-2

View this article at: https://dx.doi.org/10.21037/gist-21-2

\section{Introduction}

Gastrointestinal stromal tumors (GIST) is the most common malignant neoplasm of the gastrointestinal tract of mesenchymal origin. It develops mainly in the gastrointestinal wall - most often it is in the stomach, then in the small intestine, rectum, and esophagus. The actual incidence of GIST is difficult to determine and may range from 4.3 to as many as 22 cases per 1 million inhabitants per year (1). The basic method of treatment is surgical therapy which in some cases is combined with molecular targeted therapy. We present the following article in accordance with the narrative review reporting checklist (available at https:// dx.doi.org/10.21037/gist-21-2).

\section{Methods}

The review was conducted according to the ESMO and NCCN guidelines. The online database-PubMed, was searched using the following formula of keywords: gastrointestinal stromal tumor, surgery, laparoscopy, robotic surgery, combined therapy. Only full-text publications in English were included. There were no limits on the date of included publications. Included papers were original reports and original articles. 
Table 1 Principles of surgery in primary, resectable GIST

Complete, gross resection with negative margins (R0) with adherent organs (if necessary)-mainly wedge resections; margins 1-2 cm usually recommended

Avoiding tumor rupture

Laparoscopy/robotic surgery discouraged in larger tumors

Lymph node dissection usually not necessary

Careful exploration of the abdominal cavity in terms of dissemination

Instead of mutilating surgery, neoadjuvant therapy should be considered

GIST, gastrointestinal stromal tumor.

\section{The role of surgery in the diagnosis of GIST}

According to the European Society of Medical Oncology (ESMO) guidelines, diagnostic surgery is important in small tumors of the upper gastrointestinal tract (especially smaller than $2 \mathrm{~cm}$ ) as if there is a difficulty with endoscopic biopsy and surgery may be the only option to establish the diagnosis. The other common situation is when the tumor is excised during surgery for another reason (1). In the case of small tumors the excisional biopsy is often both a diagnostic and treatment procedure. In the case of such an operation, it is very important to maintain appropriate resection margins when planning a biopsy for excising a tumor that is suspected for GIST because small tumors usually are qualified as low-risk GISTs. An appropriate microscopically radical (R0) margin will allow the patient to avoid another surgery and reduces the chances of local recurrence $(2,3)$. In small gastro-esophageal or duodenal tumors without histopathologic confirmation of the diagnosis of GIST, the serial assessment with endoscopic ultrasound is also the option and surgery can be performed only for tumors increasing in size during follow-up.

Looking through the contemporary literature, we can find an increasing number of reports of accidentally found GISTs. The studies show that approximately $0.5 \%$ of patients after the sleeve gastrectomy are diagnosed with GIST in the postoperative material, taking into account the increasing popularity of bariatric surgery, it can be assumed that the number of patients with incidental diagnosed stromal tumors will increase (4). Another operation in which it is possible to diagnose a stromal tumor is a partial or complete gastrointestinal resection due to bleeding as a consequence of incidentally found a tumor that shows clinical symptoms and requires emergency surgery without a diagnosis (5). What is also interesting Kawanowa describes that they found GIST in 35 surgical specimens after series of 100 gastrectomies due to gastric cancer (6). Microscopic GIST can be also found in $10 \%$ of patients undergoing surgery for esophageal carcinoma (7).

Although endoscopy with biopsy is undoubtedly the gold standard in the diagnosis of GISTs, the surgical approaches should be is applicable as the curative procedure in almost all cases of GIST. Endoscopic resection may be alternative to conventional resections (full-thickness laparoscopic or with open surgery) only for small tumors when minimizing morbidity is necessary due to patients' comorbidities. Despite this, surgery remains one of the important diagnostics and therapeutic methods for small tumors accidentally excised during operation for another reason and its significance is supported by international guidelines (1).

\section{Surgical treatment of primary GIST}

The most effective method of treating primary GIST of the gastrointestinal tract (regardless of the location of the primary tumor) is a radical surgical treatment with macroscopic and microscopic negative margins to prevent local recurrence. The recommended approach is $\mathrm{R} 0$ resection with margins of about $1-2 \mathrm{~cm}(1,2)$ (Table 1 ). The published articles highlight that resection with microscopically negative margins increases chances of better survival without relapse, although this effect at lowrisk tumors is uncertain, as their impact on overall survival. In the case of primary non-radical resection with a positive evaluation of the histopathological margins (R1 resection), an experienced pathologist should re-evaluate to rule out false-positive results based on the improper orientation of specimen, tissue contraction, or encapsulation after resection (8). It is necessary to underline that GIST is predominantly growing under the mucosa layer and mucosal margins from the lumen of the gastrointestinal tract is usually not clinically meaningful (and ulcerated), similarly from the side of the peritoneal cavity the pseudocapsule of the tumor with one layer of serosa membrane may constitute the adequate margin. The decision to reoperate should be taken by a multidisciplinary team and be individualized, considering the balance between the possible risk of reoperation, the benefit to the primary tumor, and aggressiveness. Generally, re-operation after R1 resection is not routinely recommended. During operations, it is recommended to prepare the tissues gently due to the risk 
Table 2 Laparoscopic/robotic surgery of primary GIST

The use of laparoscopic/robotic-assisted resection continues to expand (no size cut-off)

Standard oncological principles are used

The goal is complete macroscopic resection and avoidance of tumor rupture; extra care while manipulating because GISTs are soft and fragile and intraperitoneal seeding is associated with a very high risk of recurrence

Use of extraction bag recommended

Limited data for non-gastric GISTs

Technically demanding

GIST, gastrointestinal stromal tumor.

of damage to the tumor capsule and the spread of neoplastic cells which leads to worsening of the patient's prognosis. Tumor infiltration into adjacent organs is not characteristic of GIST, however, in the case of difficulties in tissue preparation and the risk of tumor rupture intraoperatively, en bloc resection with adjacent organs should be considered. The risk of disease relapse after $\mathrm{R} 1$ resection is closely related to tumor rupture (9). Tumor rupture (spontaneous or iatrogenic) is an additional potent risk factor strongly associated with the increased rates of relapses. The definitions of tumor rupture have been recently developed and not all minor defects of the integrity of tumor should be classified as rupture including R1 resection or iatrogenic damage limited only to the peritoneal lining/superficial tumor capsule laceration $(10,11)$.

Lymphadenectomy is not necessary as lymphatic invasion has not usually been reported, it should be reserved only for clinically positive nodes as it may be reported in pediatric GIST $(2,12)$. The method of surgical treatment depends primarily on the location, size of the primary tumor, and mitotic index. Depending on the experience of operators and the equipment of the medical center, open procedures, as well as laparoscopic and robotic procedures may be performed (Table 2) $(2,12,13)$.

In case when R0 surgery is related to major functional sequelae or R0 surgery in localized GIST is unfeasible or may be achieved by less mutilating surgery (e.g., local resection instead of abdominal-perineal resection, local excision instead of Whipple procedure or wedge resection instead of total gastrectomy with splenectomy) and with less probability of tumor rupture or extensive blood loss, and the mutation is sensitive, preoperative treatment with imatinib should always be considered should be the rule. In this clinical situation, careful and early assessment of tumor response should be performed and surgery is usually carried out after maximal tumor response to neoadjuvant therapy (most often after 6-12 months). Imatinib is generally stopped safely one day before surgery and reassumed as soon as postoperative oral food intake is restored. Neoadjuvant strategy is especially attractive in surgically demanding tumor sites (e.g., distal rectum, gastroesophageal junction, duodenum, or esophagus), where preservation of vital functions is crucial and those patients may benefit from tumor downstaging before ultimate operation (14-18).

\section{Esophagus}

GIST of the esophagus is an extremely rare location of this tumor, therefore it is difficult to provide general recommendations for surgical treatment. Any large series and outcomes of the surgery of esophageal GIST have not been published. It should be mentioned that this location of GIST is related to a high number of recurrences and the mortality/morbidity of the surgical procedures. Due to the specific anatomical structure of esophagus segmental esophageal resections or wedge resections are not usually performed. Mainly there are two therapeutic options - surgical tumor enucleations or highly invasive esophagectomy. It is difficult to clearly define the indications for each procedure, however, it is recognized that enucleation should be performed in small tumors and esophagectomy for large and high-risk tumors. Considering the complications and mortality in the surgical treatment of the esophagus, as well as frequently coexisting diseases, the enucleation procedure may be a better therapeutic option. Enucleation of esophageal GIST can be performed in the case of smaller tumors $(2-5 \mathrm{~cm})$, while esophagectomy is recommended for GISTs greater than 9 $\mathrm{cm}$. The oncological outcomes are similar after appropriate patient selection $(19,20)$. During esophagectomy blunt or blind delamination should be minimized because it will not reliably cover maintenance of a thin potential pleural barrier that may be outside the mucosa bump. Additionally, poor tumor integrity and lack of esophageal serosa increase the risk of rupture of the tumor with a blunt preparation. Transhiatal esophagectomy would likely affect the tumors distal and middle esophagus that extend beyond the muscle and cannot be recommended. Transthoracic en bloc resection of the pleura covering the esophagus and all surrounding tissues involved, including the diaphragm, it may be recommended to avoid micro- or macroscopically incomplete resection. Left thoracoabdominal area 
the approach is recommended for larger tumors gastroesophageal connection as this will allow for perfect visualization of periapical tissue. That should make it easier en bloc resection of pleura and peritoneum tumor and diaphragm surrounding the tumor when necessary, during a break. Due to the potentially high morbidity of the esophagus and the relative lack of a significant barrier for local dilatation that hinders complete resection, imatinib should be regarded as a neoadjuvant treatment therapy for larger tumors (21).

\section{Stomach}

The most common gastric GIST procedure is a wedge or a segmental resection in the case of favorable tumor localization. In the case of large endophytic tumors, a gastrotomy may be necessary. Gastrotomy is performed proximally or distal with a normal mucosa margin about half the length of the tumor circumference. The bump is then folded over the stomach and gastrotomy as well the normal mucosa attached to the tumor is sutured in the right direction. To prevent stenosis after resection, the direction of the sutures or staplers should be perpendicular to the long axis stomach. GISTs near the GE junction may be a challenge due to the risk of stenosis. In difficult cases e.g., tumors near the GE junction, the open method should be considered. With the development of minimally invasive techniques, operations in unfavorable/anatomically difficult locations are more often performed laparoscopically and/ or endoscopically and are now the standard method of treatment in experienced centers $(22,23)$. When the tumor is near the pylorus, a distal gastrectomy may be recommended. If the stenosis is found after intraoperative suturing, additional gastrojejunostomy and vagotomy may be a rescue procedure. The choice of the surgical method of gastric GIST cannot be determined only by the size of the tumor. Open surgery may be recommended even in the case of smaller tumors if they are highly vascularized and very fragile due to the risk of rupture. Enucleation or shellout methods are not safe because they can cause peritoneal spread in consequence of pseudocapsule damage and gastric wall perforation, even in small tumors $(24,25)$.

\section{Duodenum}

Tumors bigger than $2 \mathrm{~cm}$ should be resected surgically. Due to the fact of low incidence, lack of experience, and complex anatomy of the duodeno-pancreatic area individually surgical approach is recommended.
R0 resection with 1-2 $\mathrm{cm}$ clear margin is sufficient treatment. Three main surgical options are available: pancreatoduodenectomy (PD), wedge, and limited resection (LR) defined as segmental duodenal resection (26). PD is recommended in cases involving large duodenal wart, pancreas, or duodenal pancreas wall. In clinical practice, $20 \%$ to $86 \%$ of duodenal GISTs have been reported to be treated with PD. PD is recommended for large tumors located on the medial wall of the first duodenal portion in close contact with the pancreatic head. The decision to perform a PD in patients with GISTs of the second duodenal portion is clearly influenced by the relationships of the tumor with the pancreatic head and the papilla of Vater. Large or even small tumors located on the medial wall of the second duodenal portion in close contact with the pancreatic head should be treated by PD. Large tumors located on the medial wall of the third duodenal portions and in close contact with the pancreatic head or infiltrating the pancreas should be treated with PD as well. GISTs located on the fourth duodenal portion may be locally resected and the intestinal transit restored by a primary end-to-end or end-to-side anastomosis between the third duodenal portion and jejunum. Small tumors could be treated with wedge resection (27). LR is a feasible and effective surgical treatment for patients with small-sized and antimesenteric-sided duodenal GIST in terms of late complications and postoperative diabetic complications. Minimally invasive limited resection (MILR) has better perioperative outcomes than open LR. Therefore, we should consider MI-LR as an optimal surgical treatment for selected patients with duodenal GIST. The patients in the LR group had fewer late complications than in the PD group and no postoperative newly developed diabetes mellitus. The MI-LR group had a shorter duration of surgery and a shorter length of postoperative hospital stay (28).

\section{Small intestine}

LR define as segmental resection of the small intestine with GISTs is usually recommended (29). For patients with a postoperative positive resection margin (R1) after the complete resection of primary localized GISTs (R0 resection), the NCCN guidelines do not recommend reoperation, whereas the ESMO guidelines recommend considering reoperation to achieve $\mathrm{R} 0$ resection if the original site of the lesion can be found, and no postoperative complications are expected $(1,30)$. 

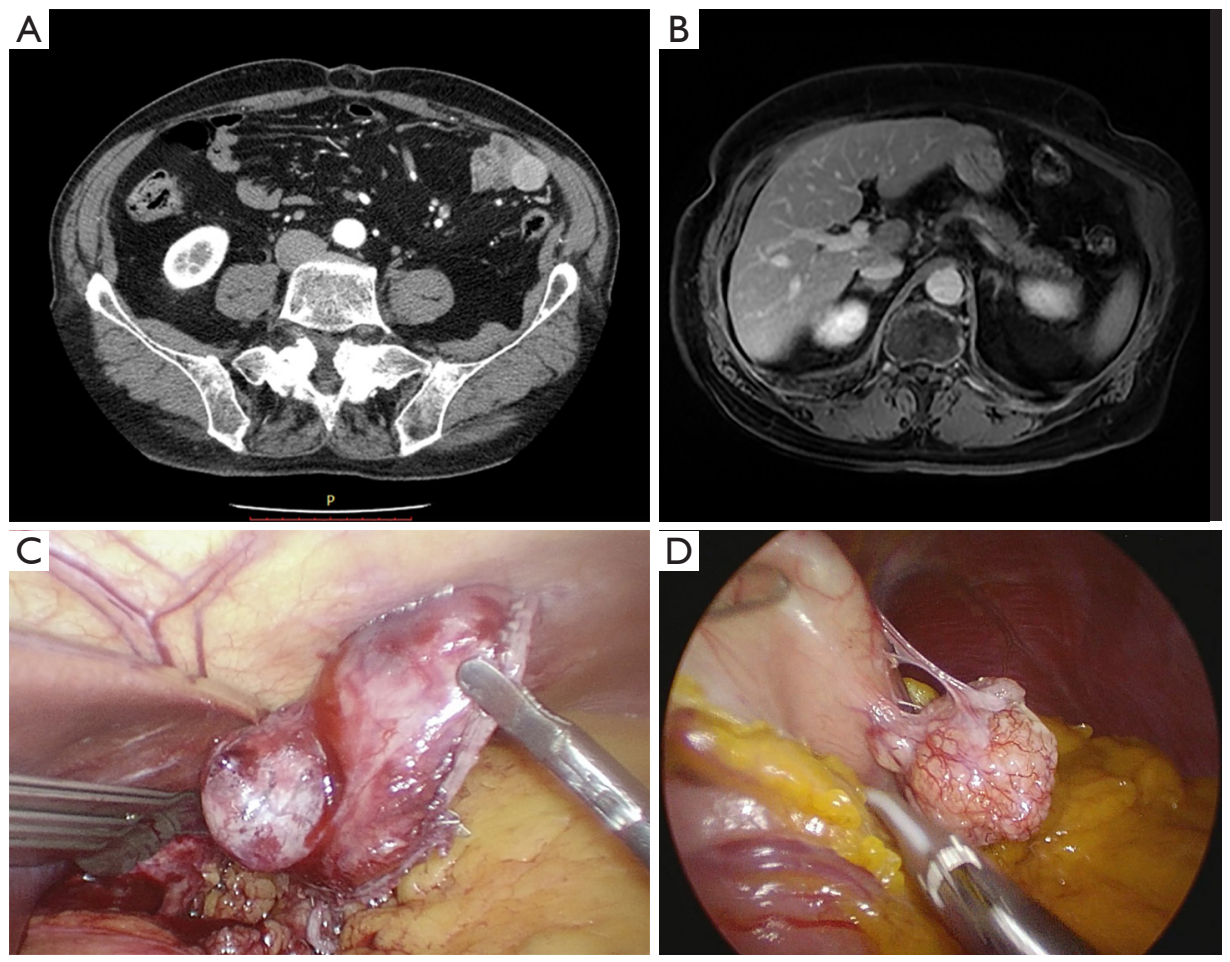

Figure 1 Two cases of GIST patients (computed tomography imaging) who underwent laparoscopic resection: (A) segmental resection of the small bowel, (B) wedge resection of the stomach, (C,D) intraoperative picture of laparoscopic resection of GIST. GIST, gastrointestinal stromal tumor.

\section{Colorectum}

The sigmoid colon is the commonest site of GIST of the large bowel, followed by a transverse colon, cecum, descending, and ascending colon. If only adjacent organs are not infiltrated an appropriate segmental resection is sufficient for surgical treatment of colonic GISTs (31).

The current treatment of rectal GIST is still controversial and difficult (32). Anatomical localization of the rectum in the pelvis, the complexity of the sphincter represents a difficulty in local management. For very low-risk, low-risk tumors, local tumor resection (LTR) or sphincter preservation may be performed. Various surgical techniques have been described for the treatment of rectal GIST, including traditional transanal resection, trans-sacral approach, transanal endoscopic microsurgery (TEM), transanal minimal invasive surgery (TAMIS), and laparoscopic surgery $(33,34)$. Conservative methods such as transvaginal (Kraske) for posterior inferior rectal GIST, and transvaginal or transdermal access in women with fewer tumors on the anterior rectal wall have been described (35).

GIST of the rectum with intestinal obstruction emergency is an exceedingly rare situation. In this case, a diverting colostomy with a biopsy of the tumor should be performed (36).

\section{En bloc GIST resection of primary GIST}

The majority of primary GIST resections are elective procedures with resections limited to one organ. However, in case of acute symptoms e.g., bleeding or gastrointestinal obstruction, extensive surgical treatment may be necessary, if the patient is enough fit for such surgery. GISTs rarely infiltrate adjacent organs. Gastric GISTs may rarely require partial gastrectomy, splenectomy, and distal pancreatectomy. Yang et al. have published cases that required en bloc multiple resections, one with partial colectomy and the other with distal pancreatectomy and splenectomy. Another case report, published in 2016, described en bloc GIST resection with the spleen and part of the diaphragm (37). In 2018, Mohamed and all published a case of en bloc resection including total gastrectomy, esophagojejunostomy, and feeding jejunostomy, distal pancreatectomy, and splenectomy. In this case, the operation was unavoidable due to its complex nature and size tumor $(42.0 \mathrm{~cm} \times$ $31.0 \mathrm{~cm} \times 23.0 \mathrm{~cm}$ in dimensions), and above all symptoms 
of gastrointestinal obstruction (38).

\section{New techniques of GIST treatment}

It is well known that new surgical technologies e.g., laparoscopic and robotic surgery can bring many benefits to the patient, compared to open surgery in surgical treatment, including the treatment of GIST e.g., faster postoperative recovery, less blood loss, and less pain, reduction of complications and postoperative morbidity (39).

\section{Laparoscopic surgery}

Traditionally, GIST resection was done with open surgery, but more recently less invasive methods have been reported (Figure 1). In the last decade, the laparoscopic approach was successfully adopted for the treatment of GIST. Evidence of laparoscopic surgery includes primarily the treatment of gastric GIST, and guidelines for tumors of the small intestine are less well defined. Although a minimally invasive approach to duodenal tumors has been described, data are still insufficient to draw firm conclusions about laparoscopic resection of GISTs located in the duodenum. Laparoscopic surgery has been successfully reported also for resection of rectal GISTs, including anus-preserving surgery. Laparoscopic colorectal surgery is beneficial because of its minimal access trauma. Adequate visualization of deep pelvic lesions is possible. Although the data on laparoscopic surgery for rectal GISTs are limited, this approach seems feasible, especially for small tumors (40).

In 1992, Łukaszczyk et al. described the first laparoscopic GIST resection and the first laparoscopic distal gastrectomy (LDG) and Kitano et al. in 1994, respectively.

The NCCN guidelines recommend laparoscopic resection for gastric GIST for tumors up to $5 \mathrm{~cm}$ (30). De Vogelaere et al. presented the results of surgical treatment of gastric GIST $>11 \mathrm{~cm}$ without damaging the tumor capsule, however, they pointed that, when the tumor is very large or its location may require excessive manipulation, laparoscopic treatment is not recommended. Larger tumors are operated with an open approach and are often needed more extensive resection. Standard laparoscopic surgery or reduced-port surgery can be applied to lesions on the great er curvature, anterior wall, and posterior wall of the stomach $(22,41-44)$.

In laparoscopic surgery, it is more difficult to recognize tumor borders. The most common method is wedgesegmental resection the procedure performed and it is the procedure of choice. In some cases, the size and location of the tumor may be an indication for more extensive surgery, including partial or total gastrectomy. Enucleation of GIST, even if still reported in the literature, avoids achieving oncological safety in the resected margin (45).

Laparoscopy can also be useful in all cases to exclude distant metastases, therefore it is recommended to start the resection with a laparoscopic examination.

Development of minimally invasive surgery such as laparoscopic wedge resection (LWR) or laparoscopic and endoscopic cooperative surgery (LECS) has been presented so far. The first document about LECS was published by Hiki et al. in 2008 and they had proved this method as a safe procedure and especially advantageous in removing the tumor completely without excessive resection of the gastric wall. Recently, several laparoscopic approaches including laparoscopic and endoscopic cooperative surgery (LECS) have been developed for small gastric GISTs near the gastroesophageal junction and pylorus to preserve junctional functions (46).

\section{Robotic surgery}

Robotic surgery can be considered useful in certain cases of treatment of primary GIST of the gastrointestinal tract, an attractive alternative form of treatment to open and laparoscopic surgery, reducing their several technical limitations. In 2010, Buchs et al. published the first document treating cases of oncological resection of large gastric GISTs using robotic surgery. This publication drew attention to the safety and attractiveness of this selected surgical technique, with good oncological results. These conclusions were also confirmed by Desiderio et al. in a study published in 2013. The magnification of the 3D image and the precise movements of the robotic arms are believed to help reach surgical sites that required previously maximum exposure and as a goal achieve negative resection margins. In 2019, Shi et al. described a new surgical technique, referring to the combination of laparoscopic and endoscopic surgery in the treatment of GIST. This new method combined the endoscopic technique and robotic surgery and was termed third space robotic and endoscopic cooperative surgery (TS-RECS). This method requires further research due to the small number of patients included in the study. The authors of the study received smaller margins than required, but in each case, it was an R0 resection. GIST was located on the anterior wall of the stomach near the greater curvature, which was probably the reason for the shorter procedure time. An additional advantage of the procedure was low blood loss and quick recovery after the surgery. TS-RECS seems to be a promising treatment for gastric GIST $>2 \mathrm{~cm}$ but this 
method requires further research due to the small number of patients included in the study $(46,47)$.

So far, robotic surgery has found its place in the surgical treatment of e.g., GIST of the esophagus, duodenum, stomach, and small intestine $(48,49)$. The role and results of robotic surgery in esophageal GISTs are not widely published due to the small number of clinical cases (50). Robotic surgery in gastric GIST is much more common, including tumors of unfavorable location, such as the esophagus-gastric junction, less curvature of the stomach, the back wall of the stomach, or the pylorus. Few scientific reports also describe robotic resections of large GIST of the stomach $>5 \mathrm{~cm}$ in compliance with oncological surgical principles $(51,52)$.

\section{Surgical treatment of residual lesions after systemic treatment of primary unresectable/disseminated cases of GIST}

In patients with unresectable or disseminated GIST (including metastatic) at diagnosis, the basic treatment option is systemic therapy with tyrosine kinase inhibitors (TKIs). This treatment is highly effective, leading to remission of the disease in most patients. Often the degree of remission makes it possible to plan an operation that may potentially be radical. The data were available in the literature from the observation of patients in whom an attempt was made to resect residual GIST lesions after systemic treatment seems to support this procedure although not formally supported by clinical trials (53-58).

Sometimes, after the successful treatment with TKIs, the limited progression of the GIST lesion(s) occursfocal disease partly not controlled by systemic treatment. In such cases, resection of this lesion may also be considered, even in a situation where the operation does not have the potential to be radical in terms of other lesions. It should be noted here that in patients diagnosed with limited progression, other options may also be considered, such as radioablation, embolization, chemoembolization, or radiotherapy of the progressing tumor.

The third, somewhat classic indication for the application of surgery in advanced GIST is an attempt to control the symptoms of the disease that are life-threatening. Among these symptoms, the most common are bleeding and gastrointestinal obstruction.

\section{Discussion/summary}

The main objective of this review was to systematize the current guidelines of surgical diagnosis and treatment of GIST. Radical surgical treatment remains the basic method of treatment of patients with local, resectable GIST, which allows achieving a permanent remission of the disease in over $50 \%$ of cases. The assessment of the risk of disease recurrence after radical surgery is used in clinical practice to determine the prognosis and plan multimodal management of an individual patient. Moreover, at present, the role of surgery in this unit is not only limited to resection of the primary tumor but what is also significant has it is an application in diagnostic. Diagnostic surgery is important in small tumors of the upper gastrointestinal tract (especially smaller than $2 \mathrm{~cm}$ ) as there is a difficulty with endoscopic biopsy and surgery may be the only option to establish the diagnosis. The other common situation is when the tumor is excised during surgery for another reason. In surgical treatment recommended approach is $\mathrm{R} 0$ resection with margins of about $1-2 \mathrm{~cm}$. The published articles highlight that resection with microscopically negative margins increases chances of better survival without relapse, although this effect at low-risk tumors is uncertain, as their impact on overall survival. The risk of disease relapse after $\mathrm{R} 1$ resection is closely related to tumor rupture. Tumor rupture (spontaneous or iatrogenic) is an additional potent risk factor strongly associated with the increased rates of relapses. Lymphadenectomy is not necessary as lymphatic invasion has not usually been reported, it should be reserved only for clinically positive nodes as it may be reported in pediatric GIST. The method of surgical treatment depends primarily on the location, size of the primary tumor, and mitotic index. Depending on the experience of operators and the equipment of the medical center, open procedures, as well as laparoscopic and robotic procedures may be performed. Currently, after resection of primary GIST at significant risk of relapse, systemic adjuvant therapy with imatinib is the standard of care as it is associated with relapse-free survival and overall survival benefits (59). The surgical strategy in locally advanced/marginally resectable GIST with sensitive mutations is also affected by the possibility of preoperative use of imatinib to facilitate resection with microscopically clear margins as well as to decrease the extent and morbidity of the surgical procedure (14). In patients with unresectable or disseminated GIST at diagnosis, the basic treatment option is systemic therapy with TKIs. Surgical treatment of residual lesions may provide a noticeable advantage for patients after systemic treatment of primary unresectable/ disseminated cases of GIST. Permanent remission of GIST 
is probably impossible to achieve without radical surgical treatment. The provided standards are adequately specified and allow a selection of the surgical procedure for each patient. Further development of GIST surgery is likely to be associated with the development of minimally invasive surgery.

\section{Acknowledgments}

Funding: None.

\section{Footnote}

Provenance and Peer Review: This article was commissioned by the Guest Editors (Bernd Kasper and Eva Wardelmann) for the series "Gastrointestinal Stromal Tumors" published in Gastrointestinal Stromal Tumor. The article has undergone external peer review.

Reporting Checklist: The authors have completed the narrative review reporting checklist. Available at https:// dx.doi.org/10.21037/gist-21-2

Peer Review File: Available at https://dx.doi.org/10.21037/ gist-21-2

Conflicts of Interest: All authors have completed the ICMJE uniform disclosure form (available at https://dx.doi. org/10.21037/gist-21-2). The series "Gastrointestinal Stromal Tumors" was commissioned by the editorial office without any funding or sponsorship. Dr. MZ reports personal fees from NOVARTIS Oncology, grants from Pierre-Fabre, personal fees from MSD, outside the submitted work. Dr. PR reports personal fees from Novartis, grants and personal fees from BMS, personal fees from MSD, grants from Pfizer, personal fees from Pierre Fabre, personal fees from Blueprint Medicines, personal fees from Sanofi, personal fees from Merck, outside the submitted work. The authors have no other conflicts of interest to declare.

Ethical Statement: The authors are accountable for all aspects of the work in ensuring that questions related to the accuracy or integrity of any part of the work are appropriately investigated and resolved.

Open Access Statement: This is an Open Access article distributed in accordance with the Creative Commons
Attribution-NonCommercial-NoDerivs 4.0 International License (CC BY-NC-ND 4.0), which permits the noncommercial replication and distribution of the article with the strict proviso that no changes or edits are made and the original work is properly cited (including links to both the formal publication through the relevant DOI and the license). See: https://creativecommons.org/licenses/by-nc-nd/4.0/.

\section{References}

1. Casali PG, Abecassis N, Aro HT, et al. Gastrointestinal stromal tumours: ESMO-EURACAN Clinical Practice Guidelines for diagnosis, treatment and follow-up. Ann Oncol 2018;29:iv68-78.

2. Rutkowski P, Skoczylas J, Wisniewski P. Is the Surgical Margin in Gastrointestinal Stromal Tumors Different? Visc Med 2018;34:347-52.

3. Rutkowski P, Nowecki ZI, Michej W, et al. Risk criteria and prognostic factors for predicting recurrences after resection of primary gastrointestinal stromal tumor. Ann Surg Oncol 2007;14:2018-27.

4. Wang S, Wang Q, Xu L, et al. Beware Pathological Findings of the Stomach in Patients Undergoing Bariatric Surgery: a Systematic Review and Meta-analysis. Obes Surg 2021;31:337-42.

5. Zhao L, Zhao Z, Wang W, et al. Current characteristics on small intestinal stromal tumor-a case control study. Ann Palliat Med 2020;9:98-107.

6. Kawanowa K, Sakuma Y, Sakurai S, et al. High incidence of microscopic gastrointestinal stromal tumors in the stomach. Hum Pathol 2006;37:1527-35.

7. Abraham SC, Krasinskas AM, Hofstetter WL, et al. "Seedling" mesenchymal tumors (gastrointestinal stromal tumors and leiomyomas) are common incidental tumors of the esophagogastric junction. Am J Surg Pathol 2007;31:1629-35.

8. Gronchi A, Bonvalot S, Poveda Velasco A, et al. Quality of Surgery and Outcome in Localized Gastrointestinal Stromal Tumors Treated Within an International Intergroup Randomized Clinical Trial of Adjuvant Imatinib. JAMA Surg 2020;155:e200397.

9. McCarter MD, Antonescu CR, Ballman KV, et al. Microscopically positive margins for primary gastrointestinal stromal tumors: analysis of risk factors and tumor recurrence. J Am Coll Surg 2012;215:53-9; discussion 59-60.

10. Hølmebakk T, Bjerkehagen B, Boye K, et al. Definition and clinical significance of tumour rupture in 
gastrointestinal stromal tumours of the small intestine. $\mathrm{Br}$ J Surg 2016;103:684-91.

11. Hølmebakk T, Hompland I, Bjerkehagen B, et al. Recurrence-Free Survival After Resection of Gastric Gastrointestinal Stromal Tumors Classified According to a Strict Definition of Tumor Rupture: A Population-Based Study. Ann Surg Oncol 2018;25:1133-9.

12. Madhavan A, Phillips AW, Donohoe CL, et al. Surgical Management of Gastric Gastrointestinal Stromal Tumours: Comparison of Outcomes for Local and Radical Resection. Gastroenterol Res Pract 2018;2018:2140253.

13. Demetri GD, Benjamin RS, Blanke CD, et al. NCCN Task Force report: management of patients with gastrointestinal stromal tumor (GIST)--update of the NCCN clinical practice guidelines. J Natl Compr Canc Netw 2007;5 Suppl 2:S1-29; quiz S30.

14. Rutkowski P, Gronchi A, Hohenberger P, et al. Neoadjuvant imatinib in locally advanced gastrointestinal stromal tumors (GIST): the EORTC STBSG experience. Ann Surg Oncol 2013;20:2937-43.

15. Wang D, Zhang Q, Blanke CD, et al. Phase II trial of neoadjuvant/adjuvant imatinib mesylate for advanced primary and metastatic/recurrent operable gastrointestinal stromal tumors: long-term follow-up results of Radiation Therapy Oncology Group 0132. Ann Surg Oncol 2012;19:1074-80.

16. Hohenberger H, Langer C, Wendtner CM, et al. Neoadjuvant treatment of locally advanced GIST: Results of APOLLON, a prospective, open label phase II study in KIT- or PDGFRA-positive tumors. J Clin Oncol 2012;30:10031.

17. Jakob J, Hohenberger P. Neoadjuvant Therapy to Downstage the Extent of Resection of Gastrointestinal Stromal Tumors. Visc Med 2018;34:359-65.

18. Kurokawa Y, Yang HK, Cho H, et al. Phase II study of neoadjuvant imatinib in large gastrointestinal stromal tumours of the stomach. Br J Cancer 2017;117:25-32.

19. Hihara J, Mukaida H, Hirabayashi N. Gastrointestinal stromal tumor of the esophagus: current issues of diagnosis, surgery and drug therapy. Transl Gastroenterol Hepatol 2018;3:6.

20. Lee HJ, Park SI, Kim DK, et al. Surgical resection of esophageal gastrointestinal stromal tumors. Ann Thorac Surg 2009;87:1569-71.

21. Blum MG, Bilimoria KY, Wayne JD, et al. Surgical considerations for the management and resection of esophageal gastrointestinal stromal tumors. Ann Thorac Surg 2007;84:1717-23.
22. Xiong $W$, Zhu J, Zheng $\mathrm{Y}$, et al. Laparoscopic resection for gastrointestinal stromal tumors in esophagogastric junction (EGJ): how to protect the EGJ. Surg Endosc 2018;32:983-9.

23. Liao GQ, Chen T, Qi XL, et al. Laparoscopic management of gastric gastrointestinal stromal tumors: A retrospective 10-year single-center experience. World J Gastroenterol 2017;23:3522-9.

24. Kong SH, Yang HK. Surgical treatment of gastric gastrointestinal stromal tumor. J Gastric Cancer 2013;13:3-18.

25. Lim KT. Surgical treatment of gastrointestinal stromal tumors of the stomach: current status and future perspective. Transl Gastroenterol Hepatol 2017;2:104.

26. Colombo C, Ronellenfitsch U, Yuxin Z, et al. Clinical, pathological and surgical characteristics of duodenal gastrointestinal stromal tumor and their influence on survival: a multi-center study. Ann Surg Oncol 2012;19:3361-7.

27. Popivanov G, Tabakov M, Mantese G, et al. Surgical treatment of gastrointestinal stromal tumors of the duodenum: a literature review. Transl Gastroenterol Hepatol 2018;3:71.

28. Lu C, Jin W, Mou Y, et al. Optimal Laparoscopic Management and Oncological Outcomes of Gastrointestinal Stromal Tumors in Duodenum: Pancreaticoduodenectomy or Pancreas-Sparing Duodenectomy? Cancer Manag Res 2020;12:4725-34.

29. Peng F, Liu Y. Gastrointestinal Stromal Tumors of the Small Intestine: Progress in Diagnosis and Treatment Research. Cancer Manag Res 2020;12:3877-89.

30. von Mehren M, Kane JM, Bui MM, et al. NCCN Guidelines Insights: Soft Tissue Sarcoma, Version 1.2021. J Natl Compr Canc Netw 2020;18:1604-12.

31. Feng F, Tian Y, Liu Z, et al. Clinicopathological features and prognosis of colonic gastrointestinal stromal tumors: evaluation of a pooled case series. Oncotarget 2016;7:40735-45.

32. IJzerman NS, Mohammadi M, Tzanis D, et al. Quality of treatment and surgical approach for rectal gastrointestinal stromal tumour (GIST) in a large European cohort. Eur J Surg Oncol 2020;46:1124-30.

33. Yang Z, Guo W, Huang R, et al. Transanal versus nontransanal surgery for the treatment of primary rectal gastrointestinal stromal tumors: a 10-year experience in a high-volume center. Ann Transl Med 2020;8:201.

34. Nepal P, Mori S, Kita Y, et al. Management of a case of high-risk gastrointestinal stromal tumor in rectum by 
transanal minimal invasive surgery. World J Surg Oncol 2018;16:165.

35. Eldamshety O, Metwally IH, Ghoneem E, et al. Resection of rectal GIST using a novel technique: a report of two cases. Ecancermedicalscience 2017;11:760.

36. Rivera AKU, Jabiles AG, Passiuri IC, et al. Gastrointestinal stromal tumour of the rectum and intestinal obstruction: case report. Ecancermedicalscience 2020;14:1139.

37. Kosmidis CS, Alexandrou V, Koimtzis GD, et al. Treatment of a Gastrointestinal Stromal Tumor (GIST) Adherent to the Spleen and the Tail of the Pancreas: A Case Report. Am J Case Rep 2020;21:e918278.

38. Mohamed A, Botros Y, Hanna P, et al. Gigantic GIST: A Case of the Largest Gastrointestinal Stromal Tumor Found to Date. Case Rep Surg 2018;2018:6170861.

39. Kim JJ, Lim JY, Nguyen SQ. Laparoscopic resection of gastrointestinal stromal tumors: Does laparoscopic surgery provide an adequate oncologic resection? World J Gastrointest Endosc 2017;9:448-55.

40. Kameyama H, Kanda T, Tajima Y, et al. Management of rectal gastrointestinal stromal tumor. Transl Gastroenterol Hepatol 2018;3:8.

41. Hu J, Or BH, Hu K, et al. Comparison of the postoperative outcomes and survival of laparoscopic versus open resections for gastric gastrointestinal stromal tumors: A multi-center prospective cohort study. Int J Surg 2016;33 Pt A:65-71.

42. De Vogelaere K, Hoorens A, Haentjens P, et al. Laparoscopic versus open resection of gastrointestinal stromal tumors of the stomach. Surg Endosc 2013;27:1546-54.

43. Kim KH, Kim MC, Jung GJ, et al. Long term survival results for gastric GIST: is laparoscopic surgery for large gastric GIST feasible? World J Surg Oncol 2012;10:230.

44. Ronellenfitsch U, Hohenberger P. Surgery for Gastrointestinal Stromal Tumors: State of the Art of Laparoscopic Resection and Surgery for M1 Tumors. Visc Med 2018;34:367-74.

45. Catena F, Di Battista M, Fusaroli P, et al. Laparoscopic treatment of gastric GIST: report of 21 cases and literature's review. J Gastrointest Surg 2008;12:561-8.

46. Shi F, Li Y, Pan Y, et al. Clinical feasibility and safety of third space robotic and endoscopic cooperative surgery for gastric gastrointestinal stromal tumors dissection : A new surgical technique for treating gastric GISTs. Surg Endosc 2019;33:4192-200.

47. Vicente E, Quijano Y, Ielpo B, et al. Robot-assisted resection of gastrointestinal stromal tumors (GIST): a single center case series and literature review. Int J Med Robot 2016;12:718-23.

48. Marano A, Allisiardi F, Perino E, et al. Robotic Treatment for Large Duodenal Gastrointestinal Stromal Tumor. Ann Surg Oncol 2020;27:1101-2.

49. Oh MH, Lee BC. Robot-assisted resection of GIST in the proximal jejunum. J Surg Case Rep 2020;2020:rjaa301.

50. Herbert B, McGinn JT, Maloney A, et al. Robotic/ thoracoscopic approach to esophageal gastro-intestinal stromal tumor. Shanghai Chest 2020;4:31.

51. Arseneaux M, Yarbrough D, Nagamoto T. Roboticassisted free-handed, full-thickness gastric GIST resection with primary repair in unfavorable locations. J Robot Surg 2019;13:491-4.

52. Desiderio J, Trastulli S, Cirocchi R, et al. Robotic gastric resection of large gastrointestinal stromal tumors. Int J Surg 2013;11:191-6.

53. Ford SJ, Gronchi A. Indications for surgery in advanced/ metastatic GIST. Eur J Cancer 2016;63:154-67.

54. Mussi C, Ronellenfitsch U, Jakob J, et al. Post-imatinib surgery in advanced/metastatic GIST: is it worthwhile in all patients? Ann Oncol 2010;21:403-8.

55. Bauer S, Rutkowski P, Hohenberger P, et al. Longterm follow-up of patients with GIST undergoing metastasectomy in the era of imatinib -- analysis of prognostic factors (EORTC-STBSG collaborative study). Eur J Surg Oncol 2014;40:412-9.

56. Rutkowski P, Hompes D. Combined Therapy of Gastrointestinal Stromal Tumors. Surg Oncol Clin N Am 2016;25:735-59.

57. Raut CP, Posner M, Desai J, et al. Surgical management of advanced gastrointestinal stromal tumors after treatment with targeted systemic therapy using kinase inhibitors. J Clin Oncol 2006;24:2325-31.

58. Fairweather M, Balachandran VP, Li GZ, et al. Cytoreductive Surgery for Metastatic Gastrointestinal Stromal Tumors Treated With Tyrosine Kinase Inhibitors: A 2-institutional Analysis. Ann Surg 2018;268:296-302.

59. Joensuu H, Eriksson M, Sundby Hall K, et al. One vs three years of adjuvant imatinib for operable gastrointestinal stromal tumor: a randomized trial. JAMA 2012;307:1265-72.

doi: $10.21037 /$ gist-21-2

Cite this article as: Kalinowska I, Zdzienicki M, Skoczylas J, Rutkowski P. A narrative review of surgical management of gastrointestinal stromal tumors. Gastrointest Stromal Tumor 2021;4:5. 